\title{
Parenting a child with phenylketonuria or galactosemia: implications for health-related quality of life
}

\author{
Amber E. ten Hoedt • Heleen Maurice-Stam • Carolien C. A. Boelen • \\ M. Estela Rubio-Gozalbo • Francjan J. van Spronsen • Frits A. Wijburg • \\ Annet M. Bosch • Martha A. Grootenhuis
}

Received: 4 July 2010 /Revised: 26 October 2010 /Accepted: 23 December 2010 / Published online: 3 February 2011

(C) The Author(s) 2011. This article is published with open access at Springerlink.com

\begin{abstract}
Parents of children with chronic disorders have an impaired health-related quality of life (HRQoL) compared to parents of healthy children. Remarkably, parents of children with a metabolic disorder reported an even lower HRQoL than parents of children with other chronic disorders. Possibly, the uncertainty about the course of the disease and the limited life expectancy in many metabolic disorders are important factors in the low parental HRQoL. Therefore, we performed a crosssectional study in parents of children with phenylketonuria (PKU, OMIM \#261600) and galactosemia (OMIM \#230400), metabolic disorders not affecting life expectancy, in order to investigate their HRQoL compared to parents of healthy
\end{abstract}

Communicated by: Peter Burgard

Competing interest: None declared.

A. E. ten Hoedt · F. A. Wijburg · A. M. Bosch $(\bowtie)$

Department of Pediatrics (H7-270), Academic Medical Center, University of Amsterdam,

Meibergdreef 9,

1105 AZ Amsterdam, The Netherlands

e-mail: a.m.bosch@amc.uva.nl

H. Maurice-Stam • M. A. Grootenhuis

Pediatric Psychosocial Department, Academic Medical Center, University of Amsterdam,

Amsterdam, The Netherlands

C. C. A. Boelen

Department of Pediatrics, Leiden University Medical Center, Leiden, The Netherlands

M. E. Rubio-Gozalbo

Department of Pediatrics and Laboratory Genetic Metabolic

Diseases, Maastricht University Medical Center,

Maastricht, The Netherlands

F. J. van Spronsen

Department of Pediatrics, University Medical Center Groningen,

Groningen, The Netherlands children and to parents of children with other metabolic disorders. A total of 185 parents of children with PKU and galactosemia aged 1-19 years completed two questionnaires. Parents of children with PKU or galactosemia reported a HRQoL comparable to parents of healthy children and a significantly better HRQoL than parents of children with other metabolic disorders. Important predictors for parental mental HRQoL were the psychosocial factors emotional support and loss of friendship. As parental mental functioning influences the health, development and adjustment of their children, it is important that treating physicians also pay attention to the wellbeing of the parents. The insight that emotional support and loss of friendship influence the HRQoL of the parents enables treating physicians to provide better support for these parents.

\section{Introduction}

In recent years, it has been demonstrated that parenting a chronically ill child affects the quality of life of the caregivers (Arafa et al. 2008; Klassen et al. 2008; Lawoko and Soares 2003). Health-related quality of life (HRQoL) can be viewed as the impact of illness and treatment on the domains of physical, psychological, social and somatic functioning and well-being (Revicki et al. 2000). Hatzmann et al. (2008) reported a significantly lower HRQoL in parents of children with chronic disorders compared to parents of healthy children in the Netherlands. Within this group of parents of chronically ill children, parents of children with a metabolic disorder reported an even lower HRQoL than parents of children with other chronic diseases, such as end-stage renal disease or Duchenne muscular dystrophy. Psychosocial determinants, like loss of friendship and emotional support, proved to be important 
predictors for parental HRQoL (Hatzmann et al. 2009). The children of the parents included in this previous study suffered from urea cycle defects, organic acidurias, lysosomal storage disorders and mitochondrial defects. In these metabolic disorders, there is a great variety in life expectancy and limited predictability of the course of the disease. This uncertain outcome could be the reason for the lower parental HRQoL in comparison to other chronic disorders. Phenylketonuria (PKU, OMIM \#261600) is an inborn error of metabolism caused by a deficiency of the enzyme phenylalanine hydroxylase (PAH, EC 1.14.16.1). As a result of this deficiency, the essential amino acid phenylalanine (Phe) cannot be converted to tyrosine and accumulates in the body. Untreated PKU results in severe intellectual disability and neurological abnormalities (Scriver et al. 2008). Since the instigation of newborn screening and early treatment with a strict lifelong protein-restricted diet with supplementation of amino acids, patients have an intellectual outcome within the normal range but slightly below the population mean (Pietz et al. 1998; Schmidt et al. 1996), and subtle neuropsychological deficits are reported (Moyle et al. 2007). Classical galactosemia (OMIM \#230400) is an autosomal recessive disorder of galactose metabolism, caused by a deficiency of the enzyme galactose-1-phosphate-uridyltransferase (GALT, EC 2.7.7.12). Patients present after ingestion of galactose with severe illness in the first weeks of life. With a galactoserestricted diet patients have a normal life expectancy. However, patients may still suffer long-term complications such as problems of mental development, disorders of speech, hypergonadotrophic hypogonadism and decreased bone mineral density (Bosch 2006). As in other metabolic disorders, parents play a pivotal role in the management of childhood PKU and galactosemia, and in spite of a normal life expectancy, both disorders still pose a heavy burden for the families. In order to be able to adequately support the parents of children with metabolic diseases, insight into the determinants of their HRQoL problems is necessary. We performed a cross-sectional study in parents of children with PKU and galactosemia, metabolic disorders not affecting life expectancy, in order to investigate the HRQoL compared to parents of healthy children and to parents of children with other metabolic disorders. Secondarily, we evaluated which variables predominantly affect the HRQoL in parents of children with PKU and galactosemia.

\section{Methods}

\section{Participants}

The participating medical centers invited parents of 148 patients with PKU and galactosemia. The Dutch Galactosemia Society and the Dutch PKU Society also sent out invitation letters; the exact number is not accessible because the Dutch PKU Society does not keep a registry so sent the letter to all members. It is inevitable that some of the parents received an invitation letter from the treating physician as well as from the Dutch PKU Society and Dutch Galactosemia Society. For privacy reasons, it was not possible to establish the exact number of parents in which this was the case.

Parents of children with PKU and galactosemia were invited by letter to participate via the treating physician of their child in the Academic Medical Centers of Amsterdam, Leiden, Maastricht and Groningen and via the Dutch PKU Society and the Dutch Galactosemia Society. Participants were enrolled in the study between December 2008 and February 2009. Parents of children with PKU or galactosemia aged 1-19 years, who had been diagnosed at least 1 year before and who were able to complete questionnaires in Dutch, were eligible for inclusion.

Data of parents of healthy children $(n=434)$ as well as data of parents of children with lysosomal storage diseases, organic acidurias or mitochondrial respiratory chain defects $(n=108)$ were used as reference data. These data were gathered in the Dutch Care-project (Hatzmann et al. 2008, 2009). Approval for this study was granted by the Ethical Committee of the Academic Medical Center (AMC), Amsterdam.

\section{Procedure}

Parents were invited through a letter in which they were asked to send an email stating their wish to participate to the investigator, after which both parents received a unique code. With the unique code, parents could log into a study website to complete the questionnaires. After 1 month, all parents received a reminder letter and 1 week before closure of the study website, parents received a reminder email. All questionnaires were completed anonymously and data could not be traced back to the individual parent.

\section{Measures}

\section{Health-related quality of life (HRQoL)}

HRQoL was assessed with the TNO-AZL Questionnaire for Adult's Health related Quality of Life (TAAQoL) (Bruil et al. 2004). The questionnaire measures health status problems weighted by the impact of problems on well-being on 12 multi-item scales: gross and fine motor functioning, cognitive functioning, sleep, pain, social functioning, daily activities, sexuality, vitality, positive emotions, depressive emotions, and aggressiveness. Each item consists of two parts: the first part assesses the prevalence of a health problem or limitation in the past month, the second part the emotional response to the health problem or limitation. 
Answers were scored on 4-point scales. A single score is attributed to each combination of an item assessing the prevalence of a problem or limitation and the corresponding emotional response. The scales vitality, positive emotions, depressive emotions and aggressiveness only assess the occurrence of the feelings in the past month. The raw scale scores are converted to 0-100; higher scores indicating better HRQoL. The validity and reliability of the TAAQoL were satisfactory (Bruil et al. 2004).

Overall physical and mental HRQoL were assessed by aggregation of all TAAQoL scale scores according to the algorithm described by Ware and Kosinski (2001), which leads to the so-called Physical Component Score (PCS) and Mental Component Score (MCS). The relative contribution of each TAAQoL scale to MCS and PCS was derived from principal components analysis, non-orthogonal rotation (Oblimin), based on the assumption that physical and mental HRQoL are interdependent.

The TAAQoL instrument can be downloaded at http:// www.tno.nl/content.cfm? context $=$ markten \&content $=$ pro duct\&laag1=189\&laag2=169\&item_id=1529

\section{Socio-demographic, medical and psychosocial variables}

Participating parents were requested to provide information about socio-demographic, medical and psychosocial variables through a selection of items from a questionnaire used in a previous study (Hatzmann et al. 2008). Variables were selected by two physicians involved in the care for patients with metabolic disorders, based on clinical relevance and existing literature.

The questionnaire consists of the following items:

Socio-demographic variables: parental gender, age (parent and child), parental chronic disease (yes/no), parental educational level and parental country of birth. Medical variables: disease development (improving, progressive, relapsing, stable) in the previous year, use of tube-feeding (yes/no); dependency on medical equipment (yes/no); problems with interaction (yes/ no); and hours of sleep per night (child). Also, care dependency (ranging from 0 to 8 ) was included, defined as the number of life domains on which the child needs care (physical, mobility, eating and drinking, medication use, coping with devices, entertaining, contact with other children, education).

Psychosocial variables: emotional support was defined as support from four groups of reference persons (partner, family, friends or neighbors), each scored on a 3 -point scale with the following values: $0=$ no, $1=$ more or less, $2=$ good, resulting in an overall score for emotional support from 0 to 8 ; loss of friendship since illness of child (yes/no), given up hobby since illness of child (yes/no), job adjustment due to illness of child (yes/no), and contact with fellow-sufferers (frequently/ often versus sometimes or never).

\section{Statistics}

The Statistical Package for Social Sciences (SPSS) Windows version 16.0 was used for all the analyses. Firstly, it was tested whether the four groups of parents (of children with PKU, galactosemia, other metabolic disorders and parents of healthy children) differed significantly on sociodemographic characteristics, using $\chi^{2}$ tests or analyses of variance (ANOVA). The significance level was set at 0.1 . Socio-demographic characteristics, including age of the child, were included in the analyses in order to be able to assess most accurately the effects of medical and psychosocial characteristics on HRQoL.

Secondly, ANOVAs by group and gender were conducted to test group differences on the TAAQoL scale scores. We used a significance level of 0.001 in order to compensate for multiple testing, since we made 66 pairwise comparisons. For each TAAQoL scale, pairwise group differences were performed by ANOVA with contrasts: healthy versus PKU, galactosemia, and Lyso/Organ/Mitochon; Lyso/Organ/Mitochon versus PKU and Galactosemia; and PKU versus galactosemia. It was not necessary to control for parental age because there was no effect of age on the TAAQoL domains, with the exception of Positive emotions.

Thirdly, linear mixed models analysis (Snijders and Bosker 2004) was performed to examine which socio-demographic, medical and psychosocial variables were predictive of mental HRQoL (MCS). Parents (first level) were treated as nested within children (second level). PCS could not be fitted in a linear mixed model because the residuals appeared not to be normally distributed. The linear mixed models analyses were performed separately for parents of children with PKU and parents of children with galactosemia.

Because of the large number of predictor variables in relation to the sample size, pre-selection of variables was necessary. Tube-feeding, dependency on medical equipment and given up hobby were excluded beforehand because of too small numbers in one of the categories. The other variables were selected for the final analysis if they proved to be associated with the outcome (MCS), the probability level was set at 0.2 in one of the following models, for PKU and/or galactosemia: MCS predicted by (1) socio-demographic variables, (2) medical variables or (3) psychosocial variables. All pre-selected variables were entered into the final models. The models for PKU and galactosemia were initially fitted with a random intercept. However, for the parents of children with PKU, there appeared not to be intercept variance so that the model for these parents was fitted with a fixed intercept. 
Table 1 Socio-demographic, medical and psychosocial characteristics

\begin{tabular}{|c|c|c|c|c|}
\hline & PKU & Galactosemia & Lyso, org, mitoch & Healthy \\
\hline \multicolumn{5}{|l|}{ Socio-demographic } \\
\hline \multicolumn{5}{|l|}{ Parental age $* *$} \\
\hline Mean (SD) & $40.7(6.3)$ & $42.5(6.5)$ & $41.5(7.0)$ & $43.7(5.4)$ \\
\hline Range & $28.4-57.4$ & $29.4-55.1$ & $23.8-60.1$ & $20.1-60.5$ \\
\hline$n$ & 86 & 41 & 108 & 441 \\
\hline \multicolumn{5}{|l|}{ Age child } \\
\hline Mean (SD) & $8.7(5.0)$ & $9.8(5.1)$ & $8.2(4.5)$ & \\
\hline Range & $1-19$ & $1-19$ & $1-18$ & \\
\hline$n$ & 109 & 65 & 108 & \\
\hline \multicolumn{5}{|l|}{ Parental gender: $\%(n) * *$} \\
\hline Male & $43.1(50)$ & $39.1(27)$ & $21.3(23)$ & $16.7(74)$ \\
\hline Female & $56.9(66)$ & $60.9(42)$ & $78.7(85)$ & $83.3(369)$ \\
\hline \multicolumn{5}{|l|}{ Parental educational level : $\%(n)^{\mathrm{a}}$} \\
\hline Low & $25.0(29)$ & $23.2(16)$ & $23.4(25)$ & $20.8(91)$ \\
\hline Middle & $39.7(46)$ & $33.3(23)$ & $42.1(45)$ & $38.2(167)$ \\
\hline High & $35.3(41)$ & $43.5(30)$ & $34.6(37)$ & $41.0(179)$ \\
\hline Parental country of birth (Netherlands): $\%(n) *$ & $94.0(109)$ & $85.5(59)$ & $95.4(103)$ & $86.0(381)$ \\
\hline Parental chronic disease (yes): $\%(n)$ & $7.8(9)$ & $5.8(4)$ & $12.0(13)$ & $7.7(34)$ \\
\hline \multicolumn{5}{|l|}{ Medical $^{\mathrm{b}}$} \\
\hline \multicolumn{5}{|l|}{ Disease development: \% $(n)$} \\
\hline Improving & $26.7(31)$ & $24.6(17)$ & $16.7(18)$ & \\
\hline Progressive & $1.7(2)$ & $2.9(2)$ & $31.5(34)$ & \\
\hline Relapsing & $14.7(17)$ & $13.0(9)$ & $23.1(25)$ & \\
\hline Stable & $56.9(66)$ & $59.4(41)$ & $28.7(31)$ & \\
\hline Tube-feeding (yes): \% $(n)$ & $0.9(1)$ & 0 & $25.9(28)$ & \\
\hline Dependency medical equipment (yes): \% $(n)$ & $6.0(7)$ & 0 & $25.9(28)$ & \\
\hline Problems making contact (yes): \% $(n)$ & $4.3(5)$ & $13.0(9)$ & $56.3(58)$ & \\
\hline \multicolumn{5}{|l|}{ Hours sleep per night } \\
\hline Mean (SD) & $9.7(1.5)$ & $9.4(1.7)$ & $9.0(2.7)$ & \\
\hline Range & $5-12$ & $3-13$ & $1-13$ & \\
\hline$n$ & 116 & 69 & 101 & \\
\hline \multicolumn{5}{|l|}{ Care dependency $(0-8)$} \\
\hline Mean (SD) & $1.1(1.1)$ & $2.0(1.8)$ & $5.1(2.3)$ & \\
\hline Range & $0-5$ & $0-7$ & $0-8$ & \\
\hline$n$ & 116 & 69 & 108 & \\
\hline \multicolumn{5}{|l|}{ Psychosocial (parent) ${ }^{\mathrm{b}}$} \\
\hline \multicolumn{5}{|l|}{ Emotional support $(0-8)$} \\
\hline Mean (SD) & $5.4(1.8)$ & $5.4(1.5)$ & $4.9(1.7)$ & \\
\hline Range & $0-8$ & $1-8$ & $1-8$ & \\
\hline$n$ & 116 & 69 & 108 & \\
\hline Loss of friendship (yes): $\%(n)$ & $11.2(13)$ & $20.3(14)$ & $55.6(60)$ & \\
\hline Given up hobby (yes): \% (n) & $3.4(4)$ & $10.1(7)$ & $45.3(49)$ & \\
\hline Job adjustment (yes): \% (n) & $21.6(25)$ & $34.8(24)$ & $25.9(28)$ & \\
\hline Contact fellow sufferers (frequently/often): \% $(n)$ & $12.1(14)$ & $27.5(19)$ & $38.9(42)$ & \\
\hline
\end{tabular}

Lyso lysosomal storage diseases, org organic acidurias, mitoch mitochondrial respiratory chain defects

*Groups differed significantly at $p<0.01$ according tot ANOVA or $\chi^{2}$ test by group

**Groups differed significantly at $p<0.001$ according tot ANOVA or $\chi^{2}$ test by group

${ }^{a}$ Highest level completed: Low Primary Education, Technical and Vocational Training, Lower and Middle General Secondary Education; Middle Middle Vocational Education, Higher General Secondary Education, Pre-university Education; High Higher Vocational Education, University

${ }^{\mathrm{b}}$ Differences between the groups not tested 
The standardized regression coefficients of the predictors were considered to be fixed because adding random effects did not result in improvement of the models at a significance level of 0.01 .

To facilitate interpretation of regression coefficients, all continuous scores were transformed into standard normal scores, expressing deviations from the overall mean of the concerning parents. We considered standardized regression coefficients of 0.1 as small, 0.3 as medium and 0.5 as large after Cohen (1988). For binary coded predictor variables, regression coefficients of 0.2 can be considered small, 0.5 medium and 0.8 large. Because of the explorative nature of the regression analyses, we used a significance level of 0.05 and trends $(p<0.1)$ are also reported.

\section{Results}

\section{Participants}

A total of 185 parents completed the questionnaires; 116 parents of children with PKU and 69 parents of children with galactosemia. Parents of at least 140 children with PKU or galactosemia participated. Although an exact response rate cannot be calculated, the number of patients included is $41 \%$ of the estimated total number of Dutch patients with PKU or galactosemia aged 119 years. Table 1 shows the characteristics of the parents and the children. Parental age of 58 participants was unknown because of a missing question in a part of the questionnaires.

Parental health related quality of life

According to ANOVAs by group and gender (and age), HRQoL of parents of children with PKU or galactosemia appeared not to differ from HRQoL in parents of healthy children, while parents of children with other metabolic disorders reported significantly worse $(p<0.001)$ HRQoL than the healthy control group on 9 out of the 12 TAAQoL scales (Table 2). Parents of children with PKU reported a better HRQoL than parents of children with other metabolic disorders $(p<0.001)$ in 8 out of 12 domains. Parents of children with galactosemia reported a significantly better HRQoL than parents of children with other

Table 2 Mean HRQoL scale scores (TAAQoL) and 95\% confidence intervals of caregivers of children with metabolic disorders and caregivers of healthy children

\begin{tabular}{|c|c|c|c|c|c|c|c|c|}
\hline & \multicolumn{2}{|c|}{ PKU $n=115$} & \multicolumn{2}{|c|}{ Galactosemia $n=69$} & \multicolumn{2}{|c|}{ Lyso, Org, Mitoch ${ }^{\mathrm{a}} n=108^{\mathrm{b}}$} & \multicolumn{2}{|c|}{ Healthy $n \approx 430^{\mathrm{c}}$} \\
\hline & Mean & $95 \% \mathrm{CI}$ & Mean & $95 \% \mathrm{CI}$ & Mean $^{\mathrm{d}}$ & $95 \% \mathrm{CI}$ & Mean & $95 \% \mathrm{CI}$ \\
\hline Gross motor & 96.5 & $94.7-98.3$ & & & $81.9 \mathrm{~b}$ & $77.7-86.2$ & 88.0 & $86.0-89.9$ \\
\hline Fine motor & & & 96.3 & $92.7-99.8$ & 94.1 & $91.3-96.9$ & 96.9 & $96.0-97.7$ \\
\hline Cognitive & 82.7 & $78.7-86.7$ & 81.4 & $75.1-87.8$ & $68.9 \mathrm{a}$ & $63.6-74.2$ & 78.9 & $76.5-81.2$ \\
\hline Sleep & 75.7 & $71.3-80.1$ & 59.8 & $72.6-67.7$ & $58.2 \mathrm{ab}$ & $52.7-63.7$ & 70.7 & $68.3-73.0$ \\
\hline Pain & 78.3 & $74.6-82.0$ & 74.5 & $68.7-80.2$ & $62.1 \mathrm{ab}$ & $56.8-67.4$ & 71.9 & $69.8-74.0$ \\
\hline Social & 84.1 & $80.7-87.5$ & 84.2 & $80.1-88.4$ & $70.9 \mathrm{abc}$ & $66.4-75.3$ & 83.5 & $81.6-85.4$ \\
\hline Daily activities & 87.7 & $83.7-91.7$ & 88.6 & $83.5-93.7$ & $68.3 \mathrm{abc}$ & $63.0-73.7$ & 85.3 & $83.2-87.3$ \\
\hline Sexuality & 84.6 & $80.1-89.1$ & 86.2 & $80.5-92.0$ & $70.6 \mathrm{abc}$ & $65.1-76.0$ & 84.8 & $82.5-87.0$ \\
\hline Vitality & 63.8 & $59.1-68.4$ & 62.0 & $56.9-67.0$ & $47.7 \mathrm{abc}$ & $42.7-52.7$ & 63.3 & $61.2-65.4$ \\
\hline Pos. emotions & 64.6 & $60.8-68.5$ & 62.4 & $57.9-67.0$ & $56.3 \mathrm{a}$ & $52.5-60.2$ & 66.4 & $64.5-68.3$ \\
\hline Depres. emotions & 78.9 & $75.6-82.2$ & 75.2 & $70.6-79.8$ & $60.1 \mathrm{abc}$ & $56.2-64.0$ & 78.6 & $76.8-80.4$ \\
\hline Agressiveness & 89.3 & $86.6-91.9$ & 90.2 & $87.3-93.1$ & 83.0 & $79.8-86.2$ & 86.0 & $89.1-89.6$ \\
\hline
\end{tabular}

Note: the empty boxes indicate exclusion because of Cronbach's $\alpha<0.60$

${ }^{\text {a }}$ Lyso lysosomal storage diseases, org organic acidurias, mitoch mitochondrial respiratory chain defects

${ }^{\mathrm{b}}$ For the scale Sexuality $\mathrm{N}=104$

${ }^{\mathrm{c}} n$ between 414 (Sexuality scale) and 433 (Depressive emotions scale)

$* p<0.001$

${ }^{\mathrm{d}}$ Letters in this column, all significant at $p<0.001$ :

a Lyso, Org, Mitoch versus healthy, according to ANOVA by group and gender; Positive Emotions: ANOVA by group, gender and parental age b Lyso, Org, Mitoch versus PKU, according to ANOVA by group and gender

c Lyso, Org, Mitoch versus galactosemia, according to ANOVA by group and gender 
metabolic disorders $(p<0.001)$ in 5 out of 12 domains. We did not find any difference between parents of children with PKU and galactosemia according to ANOVA at $p<0.001$.

Predictors of mental HRQoL in parents of children with PKU or galactosemia

Parameter estimates from the linear mixed model analyses of parental mental HRQoL are shown in Table 3. The size of most standardized regression coefficients was small to medium.

Mental HRQoL (MCS) in parents of children with PKU was affected significantly by the age of their child, emotional support and loss of friendship. A higher age of the child ( $\beta=$ $0.21, p<0.05)$ and more emotional support $(\beta=0.20, p<$ 0.05 ) was associated with a better MCS. Loss of friendship was negatively associated with MCS $(\beta=-1.2, p<0.001)$. In addition, a trend was found for the effect of disease development. Parents who considered the disease development as stable in the previous year showed better MCS $(\beta=$ $0.32, p=0.071$ ).
MCS in parents of children with galactosemia was only significantly affected by emotional support, which seemed positively associated with MCS $(\beta=0.38, p<0.001)$. In addition, the results indicated that mothers experienced worse MCS than fathers $(\beta=-0.38, p=0.062)$, as did parents who had adjusted their job since the illness of their child ( $\beta=-0.41, p=0.072)$. Furthermore, a stable disease in the previous year tended to be positively associated with $\operatorname{MCS}(\beta=0.38, p=0.059)$.

\section{Discussion}

In this study, we demonstrate a normal HRQoL in parents of children with PKU and galactosemia. This finding is remarkable because both these disorders cause a substantial burden on the family (Kazak et al. 1988) and the HRQoL of the children with classical galactosemia is severely hampered due to the long-term complications that are seen in this disorder (Bosch et al. 2004). As we expected, these parents do have a better outcome compared to parents of children with other metabolic disorders

Table 3 Mental component scale scores (TAAQoL) of parents of children with PKU and Galactosemia predicted by demographic, medical and psychosocial characteristics, in a multilevel linear model

\begin{tabular}{|c|c|c|c|c|c|c|}
\hline & \multicolumn{3}{|l|}{ PKU } & \multicolumn{3}{|l|}{ Galactosemia } \\
\hline & Coefficient $\beta$ & SE of $\beta$ & $95 \% \mathrm{CI}$ & Coefficient $\beta$ & SE of $\beta$ & $95 \% \mathrm{CI}$ \\
\hline \multicolumn{7}{|l|}{ Fixed effects } \\
\hline \multicolumn{7}{|l|}{ Socio-demographic } \\
\hline Age child & $0.21 *$ & 0.10 & $0.02 ; 0.41$ & -0.09 & 0.10 & $-0.29 ; 0.10$ \\
\hline Parental gender (female) & -0.17 & 0.16 & $-0.49 ; 0.15$ & $-0.38^{\mathrm{b}}$ & 0.20 & $-0.78 ; 0.02$ \\
\hline Native country (Netherlands) & 0.45 & 0.34 & $-0.22 ; 1.12$ & -0.07 & 0.29 & $-0.66 ; 0.51$ \\
\hline Chronically ill & -0.20 & 0.32 & $-0.83 ; 0.43$ & -0.17 & 0.43 & $-1.02 ; 0.68$ \\
\hline \multicolumn{7}{|l|}{ Medical } \\
\hline Disease development (stable) & $0.32^{\mathrm{a}}$ & 0.17 & $-0.03 ; 0.66$ & $0.38^{\mathrm{c}}$ & 0.20 & $-0.02 ; 0.77$ \\
\hline Care dependency & -0.17 & 0.11 & $-0.40 ; 0.05$ & -0.07 & 0.10 & $-0.26 ; 0.12$ \\
\hline \multicolumn{7}{|l|}{ Psychosocial } \\
\hline Emotional support & $0.20 *$ & 0.09 & $0.02 ; 0.38$ & $0.38^{* *}$ & 0.10 & $0.17 ; 0.58$ \\
\hline Loss of friendship & $-1.2 * *$ & 0.32 & $-1.8 ;-0.52$ & 0.06 & 0.28 & $-0.49 ; 0.62$ \\
\hline Contact fellow sufferers & 0.11 & 0.27 & $-0.41 ; 0.64$ & 0.18 & 0.22 & $-0.25 ; 0.61$ \\
\hline Job adjustment & 0.09 & 0.22 & $-0.36 ; 0.53$ & $-0.41^{\mathrm{d}}$ & 0.22 & $-0.86 ; 0.04$ \\
\hline$\%$ Explained variance by fixed effects & 31.7 & & & 30.6 & & \\
\hline Total number of observations & 109 & & & 65 & & \\
\hline
\end{tabular}

Variables not included in the model: tube-feeding, dependency on medical equipment, problems making contact, hours of sleep per night, given up hobby.

* $p<0.05,{ }^{* *} p<0.001$

${ }^{\mathrm{a}} p=0.071$

${ }^{\mathrm{b}} p=0.062$

${ }^{\mathrm{c}} p=0.059$

${ }^{\mathrm{d}} p=0.072$ 
who, in a previous study, reported a severely hampered HRQoL (Hatzmann et al. 2009). The main difference between the patient groups in the present study and the study by Hatzmann et al. (2009) is the difference in life expectancy and predictability of the course of the disease, which could well explain the differences in the HRQoL of the parents. Furthermore, the differences between the metabolic groups in terms of loss of friendship and giving up hobbies as a result of the disease of the child should be considered. Even though the findings show that parents of children with PKU and galactosemia have a normal HRQoL, the potential difficulties these parents experience should not be underestimated.

The strength of this study is the high response rate which might suggest that the respondents are a reliable representation of the total of parents of children with PKU and galactosemia. However, we do not have demographic information of the non-responders, and the respondents were for the most part mothers, although a higher percentage of fathers responded compared to both the group of parents of healthy children and the group of parents of children with other metabolic disorders. To attain a more complete picture of parental HRQoL, a more thorough exploration of the HRQoL of fathers is preferable.

In our study, psychosocial determinants like emotional support and loss of friendship were predictive for parental mental HRQoL, which has been reported before in other chronic disorders including metabolic disorders (Hatzmann et al. 2008, 2009). Furthermore, a higher age of the child was associated with a better parental mental HRQoL in parents of children with PKU. There are several possible explanations for this phenomenon. Firstly, younger children tend to need more care than older children, which can be a burden to parents. Secondly, when the child is very young, parents still need to get used to the protein-restricted diet of their children, and thirdly, parents may be more uncertain about how well the child will develop and what the consequences for the future may be.

Children with chronic disorders are vulnerable to develop adjustment problems when compared to their healthy peers (Wallander and Varni 1998; Pless and Nolan 1991). Furthermore, parental mental functioning influences the health, development and adjustment of their children (Prince et al. 2007; Friedman et al. 2004). Therefore, it is important that physicians involved in the care of children with a chronic disorder also pay attention to the well-being of the parents. The insight that emotional support and loss of friendship influence the HRQoL of parents of children with PKU and galactosemia enables treating physicians to provide parents adequate support. Parents need to be advised to be on the alert for loss of friendships and emotional support and to maintain their social network, as this might have consequences for their HRQoL. Parents at risk for HRQoL impairment should be referred to social work and psychosocial care to take a survey of the problems and to empower parents to set up or maintain an adequate support system.

Acknowledgments This study has been financially supported by the Dutch PKU Society and the Dutch Galactosemia Society. The authors confirm independence from the sponsors; the content of the article has not been influenced by the sponsors.

Open Access This article is distributed under the terms of the Creative Commons Attribution Noncommercial License which permits any noncommercial use, distribution, and reproduction in any medium, provided the original author(s) and source are credited.

\section{References}

Arafa MA, Zaher SR, El-Dowaty AA, Moneeb DE (2008) Quality of life among parents of children with heart disease. Health Qual Life Outcomes 6:91

Bosch AM (2006) Classical galactosaemia revisited. J Inherit Metab Dis $29: 516-525$

Bosch AM, Grootenhuis MA, Bakker HD, Heijmans HS, Wijburg FA (2004) Living with classical galactosemia: health-related quality of life consequences. Pediatrics 113:e423-e428

Bruil J, Fekkes T, Vogels T, Verrips GHW (2004) TAAQOL Manual. Leiden Center for Child Health and Pediatrics LUMC-TNO

Cohen J (1988) Statistical power analysis for the behavioral sciences, 2nd edn. Erlbaum, Hillsdale

Friedman D, Holmbeck GN, Jandasek B, Zukerman J, Abad M (2004) Parent functioning in families of preadolescents with spina bifida: longitudinal implications for child adjustment. J Fam Psychol 18:609-619

Hatzmann J, Heymans HS, Ferrer-i-Carbonell A, van Praag BM, Grootenhuis MA (2008) Hidden consequences of success in pediatrics: parental health-related quality of life-results from the Care Project. Pediatrics 122:e1030-e1038

Hatzmann J, Valstar MJ, Bosch AM, Wijburg FA, Heymans HS, Grootenhuis MA (2009) Predicting health-related quality of life of parents of children with inherited metabolic diseases. Acta Paediatr 98:1205-1210

Kazak AE, Reber M, Snitzer L (1988) Childhood chronic disease and family functioning: a study of phenylketonuria. Pediatrics 81:224-230

Klassen AF, Klaassen R, Dix D, Pritchard S, Yanofsky R, O'Donnell M, Scott A, Sung L (2008) Impact of caring for a child with cancer on parents' health-related quality of life. J Clin Oncol 26:5884-5889

Lawoko S, Soares JJ (2003) Quality of life among parents of children with congenital heart disease, parents of children with other diseases and parents of healthy children. Qual Life Res 12:655-666

Moyle JJ, Fox AM, Arthur M, Bynevelt M, Burnett JR (2007) Metaanalysis of neuropsychological symptoms of adolescents and adults with PKU. Neuropsychol Rev 17:91-101

Pietz J, Dunckelmann R, Rupp A et al (1998) Neurological outcome in adult patients with early-treated phenylketonuria. Eur J Pediatr $157: 824-830$

Pless IB, Nolan T (1991) Revision, replication and neglect-research on maladjustment in chronic illness. J Child Psychol Psychiatry 32:347-365

Prince M, Patel V, Saxena S, Maj M, Maselko J, Phillips MR, Rahman A (2007) No health without mental health. Lancet 370:859-877 
Revicki DA, Osoba D, Fairclough D, Barofsky I, Berzon R, Leidy NK, Rothman M (2000) Recommendations on health-related quality of life research to support labeling and promotional claims in the United States. Qual Life Res 9:887-900

Schmidt H, Burgard P, Pietz J, Rupp A (1996) Intelligence and professional career in young adults treated early for phenylketonuria. Eur J Pediatr 155:S97-S100

Scriver R, Levy H, Donlon J (2008) Hyperphenylalaninemia: Phenylalanine hydroxylase deficiency. In the online metabolic and molecular bases of inherited disease. Retrieved from: url: www.ommbid.com

Snijders FAB, Bosker RJ (2004) Multilevel analysis. An introduction to basic and advanced multilevel modeling. Sage, London

Wallander JL, Varni JW (1998) Effects of pediatric chronic physical disorders on child and family adjustment. J Child Psychol Psychiatry 39:29-46

Ware JE, Kosinski M (2001) Interpreting SF-36 summary health measures: a response. Qual Life Res 10:405-413 